\title{
Gas permeation through a polymer network
}

\author{
B. Schmittmann ${ }^{1} \ddagger$, Manoj Gopalakrishnan ${ }^{2}$, and R. K. P. Zia ${ }^{1}$ \\ ${ }^{1}$ Physics Department and Center for Stochastic Processes in Science and Engineering, \\ Virginia Tech, Blacksburg, VA 24061-0435, USA \\ ${ }^{2}$ MPI für Physik komplexer Systeme, Nöthnitzer Str. 38, 01187 Dresden, Germany
}

\begin{abstract}
We study the diffusion of gas molecules through a two-dimensional network of polymers with the help of Monte Carlo simulations. The polymers are modeled as non-interacting random walks on the bonds of a two-dimensional square lattice, while the gas particles occupy the lattice cells. When a particle attempts to jump to a nearest-neighbor empty cell, it has to overcome an energy barrier which is determined by the number of polymer segments on the bond separating the two cells. We investigate the gas current $J$ as a function of the mean segment density $\rho$, the polymer length $\ell$ and the probability $q^{m}$ for hopping across $m$ segments. Whereas $J$ decreases monotonically with $\rho$ for fixed $\ell$, its behavior for fixed $\rho$ and increasing $\ell$ depends strongly on $q$. For small, non-zero $q, J$ appears to increase slowly with $\ell$. In contrast, for $q=0$, it is dominated by the underlying percolation problem and can be non-monotonic. We provide heuristic arguments to put these interesting phenomena into context.

PACS numbers: 05.40.Fb, 64.60.Ak, 61.41.+e
\end{abstract}

\section{Introduction}

Permeation of gas molecules through an amorphous polymer film is a problem of considerable scientific interest, as well as industrial significance [1]. For example, the selective permeability of certain types of polymeric materials is often used for gas separation. It is therefore of primary importance to understand how the diffusivity of different gases depend on various factors, such as the temperature, the size of the penetrant molecule, and the total amount and distribution of accessible free volume inside the polymer matrix [2]. In a typical gas permeation experiment, a polymer film, formed by cooling the polymer from the rubbery state below the glass transition, is subjected to a pressure gradient across the film, so that gas molecules permeate the film. After a time lag $\tau$, the pressure on the low-pressure side starts to increase, and eventually, a stationary gas current across the membrane is established. For very long times, the pressures on both sides of the film equilibrate; this late stage is not of interest here. The important parameters which are often used to describe the permation process are $(i)$ the solubility $S$, which gives the total amount of gas trapped in the membrane $\ddagger$ e-mail: schmittm@vt.edu 
and $(i i)$ the diffusion coefficient $D$ of a gas molecule inside the network. To determine the latter, one approximates the late-time dependence of the pressure by a straight line and obtains its intercept, $t_{o}$, with the zero pressure axis. The diffusion coefficient then follows from the relation $t_{o}=L^{2} / 6 D$, where $L$ is the thickness of the film. The product of the solubility and diffusion coefficient is referred to as the permeability $P=D S$, and is a direct measure of the efficiency of the permeation process.

From a slightly different point of view, the gas permeation problem may be discussed in the language of (electric) resistor networks. The pressure gradient set up across the membrane is analogous to a fixed voltage difference. Local configurational fluctuations inside the polymer matrix allow gas molecules to jump across energy barriers between voids, forming a connected set of "resistors" which control the flow of the gas current. In fact, passage of (electric) current through a set of randomly distributed resistors is a problem that has been extensively studied in statistical physics [3]. From this perspective, gas diffusion through a polymer network is quite intriguing since the matrix of energy barriers and voids can hardly be considered random or uncorrelated.

It is therefore natural to explore the role of these correlations in the transport process. The primary objective of this paper is to introduce a lattice-gas model with correlated, quenched energy barriers to study the transport of gas molecules through the polymer network. While we simplify the description significantly, by reducing it to two dimensions, and modeling polymers as simple non-interacting random walks, we nevertheless retain an essential feature of the original problem, namely, the effect of correlations induced by the polymer connectivity. We find that the currents through this network depend very sensitively on $(i)$ whether particle motion can be completely blocked by certain energy barriers; and $(i i)$ whether these spatially correlated barriers can percolate across the lattice. Our discussion draws on an earlier study [4 in which we investigated the percolation properties of random walks of varying length.

The remainder of this paper is divided into three sections: Section II contains the model description, Section III discusses the Monte Carlo simulation results and Section IV outlines our conclusions.

\section{The model}

We consider a polymer network whose large scale structure is static over the time scale of the simulations. This is a reasonable simplification since typical permeation experiments use polymers in the glassy state: Such membranes offer better selectivity than those in a rubbery state. Small local rearrangements - which let gas molecules pass from one void to another - do occur and will be modeled via a distribution of energy barriers which the molecules need to overcome. The polymer network is generated by placing $N$ non-interacting random walks of length $\ell$ (referred to as " $\ell$-mers") on a two-dimensional square lattice with $L^{2}$ sites. Each bond of the lattice can carry one or more segments (i.e., steps of the random walk), with an average segment density per bond of $\rho=N \ell / 2 L^{2}$. The gas molecules are modeled as hard-core particles which occupy the cells of the 
lattice, and no more than one particle is allowed per cell.

While modeling polymers as non-interacting (rather than self-avoiding) walks is almost certainly an oversimplification, we can offer two motivations for such a simple description. First, real membranes are produced by rapid cooling of a rubbery melt; hence, typical polymer configurations are "frozen" high-temperature states for which interactions are less important. Second, when discretizing a real network, it is natural to choose the lattice spacing to be comparable to the polymer persistence length. In this case, a polymer "segment" contains many monomers. Since the persistence length is typically much larger than the size of a monomer unit, we have in effect adopted a coarse-grained description. On this length scale, self-avoidance and other complications may be neglected.

Each polymer segment constitutes an energy barrier in the path of a gas molecule. The dynamics of the molecules is modeled as activated hopping over these barriers. Once a particle-hole pair, separated by such a barrier, is chosen, the probability for them to exchange is $q^{m}$, where $m$ is the number of segments in the barrier and $q$ is just $e^{-\beta \epsilon}$, with $\epsilon$ being an energy scale and $\beta=1 / k_{B} T$ being the inverse temperature. As an alternate view, one may consider the particle motion as resulting from the short time scale segmental motion of the polymer. If the probability of displacement of a single segment is $q$, then $q^{m}$ would be the probability of simultaneously displacing all $m$ segments.

To model the pressure gradient, the cells in the first row $(y=0)$ of the lattice are kept filled at all times. At the beginning of the simulation (time $t=0$ ), the remainder of the lattice is empty. At times $t>0$, each particle randomly selects a direction of motion with probability $1 / 4$, and attempts to cross the barrier between its originating and target cells. Provided the target cell is empty, the particle will be moved with probability $q^{m}$. After every particle in the system has attempted a move (on average), $t$ is incremented by one Monte Carlo step (MCS). When a particle arrives in a cell within the last row $(y=L)$, it is removed instantaneously from the lattice. Clearly, this sets up a density gradient across the system which drives a particle current in the $y$-direction.

In the simulations, we measure the following quantities:

(i) The number of particles which have entered the system up to time $t$, denoted by $M_{1}(t)$

(ii) The number of particles which have exited the system up to time $t$, denoted by $M_{2}(t)$.

(iii) The mean first passage time, $\tau$, for the arrival of the first particle at the far boundary $y=L$.

At any time $t$, therefore, the number of particles absorbed by the polymer network is given by $M(t)=M_{1}(t)-M_{2}(t)$. At sufficiently late times, the system approaches a steady state, which is characterized by a time-independent solubility

$$
M(\rho, \ell ; q) \equiv \lim _{t \rightarrow \infty} M(t)
$$


and a constant particle current, or permeability, $J(\rho, \ell ; q)$, defined via

$$
J(\rho, \ell ; q) \equiv \lim _{t \rightarrow \infty} \partial_{t} M_{2}(t)
$$

To give a reference point, the current through an empty lattice would be $1 / 2$. The mean first-passage time provides information about the typical path length travelled by a particle, and how quickly it hops over the barriers encountered along that path.

The numerical simulations were carried out for various values of the segment density $\rho$ and polymer length $\ell$. We choose a lattice with $128 \times 128$ sites for the simulations, which was also the largest lattice simulated in [4]. We choose polymer lengths in powers of $4: \ell=4^{n}$ with $n=0,1,2,3,4$. The typical span of even the longest polymer $(\ell=256)$ is much smaller than the lattice dimension, so that a single polymer only very rarely extends across the whole lattice. Our choices for the segment density $\rho$ were guided by the phase diagram of the $q=0$ system which we will summarize in the next section. The smallest value of $\rho$ was 0.5 and the largest was 1.0. Intermediate values (e.g. $\rho=0.71$ ) are controlled by $N$, the (integer) number of polymers of length $\ell$ placed in the system. For different $\ell$ 's, we selected $N$ 's to be within less than a percent of $2 \rho L^{2} / \ell$. So far, we considered just two values of the bond-crossing probability: $q=0.0$ and $q=0.1$. Due to computational limitations, we generated between 15 and 48 different polymer configurations for each set of parameters $(\rho, \ell, q)$. $M(t)$ and $M_{2}(t)$ are collected for $2 \times 10^{6}$ MCS for each configuration and then averaged.

Let us comment briefly on statistical errors, which can be estimated by comparing currents for different configurations at the same $(\rho, \ell, q)$. They are smallest (below 5\%) for the shortest polymers and $q=0.1$. As $\ell$ increases, the errors also become larger, reaching about $20 \%$ for $\ell=256$ at this $q$. For $q=0.0$ and especially $\rho=0.71$, finite-size effects allow for both blocked and open configurations at the same $(\rho, \ell, q)$, making it difficult to assign error bars reliably. A conservative estimate would give errors as large as the currents themselves, so that our conclusions can only be preliminary until better statistics are available.

\section{Numerical Results}

\subsection{The $q=0$ case: Percolation of occupied bonds}

When the bond-crossing probability $q$ vanishes (or, equivalently, the zero temperature limit), bonds carrying one or more segments become completely impenetrable. As a consequence, the gas current must vanish if the occupied bonds form a connected path (a "spanning cluster") transverse to the density gradient. The problem, therefore, reduces to bond percolation of $N \ell$-mers on a square lattice. We studied this problem in [4, and summarize only the salient results here.

With the segment density (per bond) given by $\rho=N \ell /\left(2 L^{2}\right)$, the fraction of occupied bonds, $p(\rho, \ell)$, approaches a well-defined value in the thermodynamic limit. In our simulations, we found that $p$ decreases with $\ell$ for fixed $\rho$ and increases with $\rho$ for

fixed $\ell$. While the latter behavior is obvious, the former needs some explanation. To 
make it more intuitive, let us consider two situations where the density $\rho$ is kept fixed, but the polymer lengths (and hence, their numbers) are different. Configurations with few, long polymers are likely to exhibit numerous bonds which are multiply occupied since a simple random walk retraces any given step with probability $1 / 4$. In contrast, such multiple bond crossings are far less likely for short polymers, even at the same $\rho$, resulting in a larger $p$. To rephrase, single segments leave far fewer bonds unoccupied ("free") than longer polymers.

With a qualitative understanding of $p(\rho, \ell)$, it is natural to ask how the percolation threshold, $p_{c}(\ell)$, is crossed as one increases $\rho$ at given $\ell$. From simulations, we find that $p_{c}(\ell)$ is a monotonically decreasing function of $\ell$, i.e., when $\ell$ is large, a smaller number of occupied bonds already suffices to ensure the presence of a spanning cluster. We believe that this is primarily due to long polymers forming extended cigar-shaped objects [5] which percolate more easily [6] than single occupied bonds or short polymers.

Clearly, two consequences of polymerization compete with one another: the elongated shape of the polymers tends to lower, while the presence of multiple occupancies tends to increase, the percolation threshold. Due to this competition, the critical segment density per bond, $\rho_{c}(\ell)$, is a non-monotonic function of $\ell$, shown in Fig. 1 for a $128 \times 128$ square lattice. We see that $\rho_{c}(\ell)$ increases from $\ln 2=0.692$ for $\ell=1$ to 0.744 for $\ell=4$ but decreases beyond that to $0.724(\ell=16)$ and 0.703 for $\ell=64$. For shorter polymers, $\rho_{c}(\ell)$ is dominated by the effect of multiple occupancies (which increase with $\ell$ ), but for longer ones, their tendency to form extended objects which percolate more easily, takes over. When $\ell$ becomes comparable to $L$, finite-size effects become noticeable, causing $\rho_{c}(\ell)$ to increase again: $\rho_{c}(256)=0.718$ for $L=128$. Here, multiple occupancies dominate again, this time due to large sections of different polymers overlapping one another. Not surprisingly, this effect is more pronounced on smaller lattices, making it difficult to extrapolate the behavior of $\rho_{c}(\ell)$. Whatever the eventual resolution of these finite-size effects, in the thermodynamic limit $\rho_{c}(\ell)$ plays the role of a phase boundary: a gas current can flow only if $\rho<\rho_{c}(\ell)$.

\subsection{The gas current $J$}

Our earlier findings 4 provide an answer to a simple "yes/no" question: Will a gas current flow or not? We now turn to a much more detailed question: If a current does flow, what are its quantitative characteristics? By measuring currents, we are probing not only the mere existence of open channels, but also their number and their length: How many distinct paths are there for a gas molecule to travel from one edge of the system to the other, and how many cells will it visit along a given path? Again, we use a combination of simulations and simple intuitive arguments to explore the answers. We begin with systems in the low temperature limit $q=0.0$, i.e., occupied bonds cannot be crossed at all, and then consider the effects of softening this constraint by having non-zero $q$.

Our numerical results for the steady-state particle current $J(\rho, \ell ; q)$ are presented 


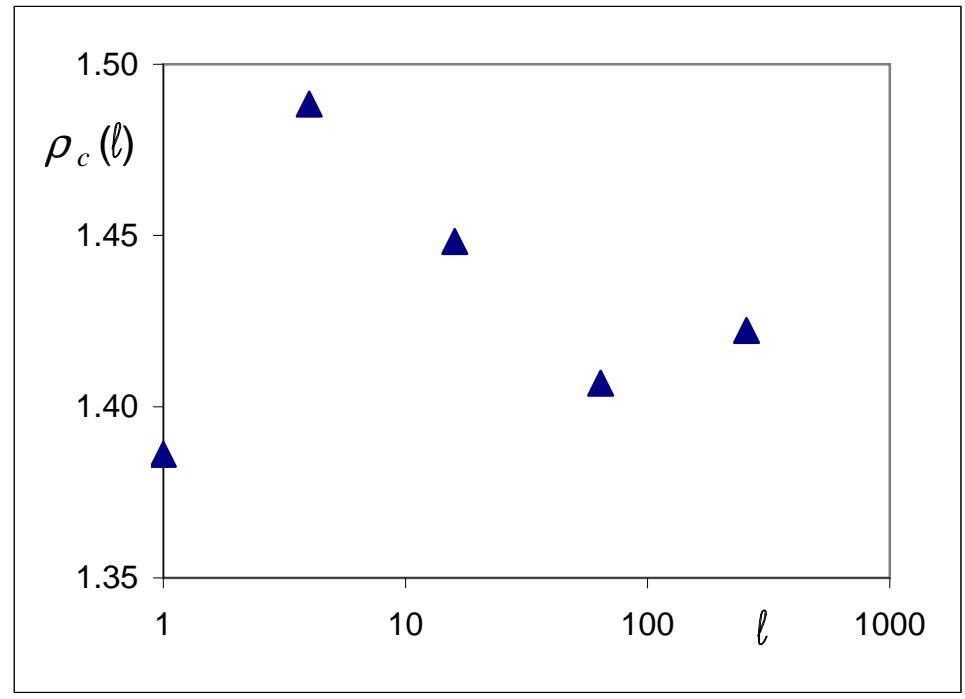

Figure 1. The critical segment density $\rho_{c}(\ell)$ vs. polymer length $\ell$ at the percolation threshold for system size $128 \times 128$ [4].

in Figs. 2 and 3. In Fig. 2, we show $J$ plotted vs. polymer length $\ell$, for fixed $\rho=0.5$ and $q=0.0$. According to the results summarized in the previous section, this value of $\rho$ is well below the percolation density $\rho_{c}(\ell)$ for all $\ell$ considered here; hence the current is non-zero for these $\ell$. In contrast, the inset shows $J$ vs. $\ell$ at a higher density, $\rho=0.71$, which is within a few percent of the critical $\rho_{c}(\ell)$. In fact, for $\ell=1, \rho$ lies slightly above the critical $\rho_{c}(1)=\ln 2$, and consequently, no current should flow for this $\ell$. As one might anticipate, the absolute values for the currents are much smaller (by at least a factor of 10) for the larger $\rho$, since more bonds are blocked in that case. On a more quantitative level, we observe very different behaviors at the two densities. For $\rho=0.5$, $J$ decreases with $\ell$, for all but the smallest $\ell$. In contrast, at $\rho=0.71$, the current first increases, then drops off again to a minimum at $\ell=64$, after which it recovers. Thus, polymerization has very different effects on the current, at low and high segment density.

We now attempt to interpret these findings, in the light of our percolation study summarized above. Considering, first, $\rho=0.5$, we note that this value is well below $\rho_{c}(\ell)$ for all $\ell$ considered. In other words, a typical configuration exhibits only finite clusters of occupied bonds, allowing for numerous current-carrying channels with a length of $O(L)$. Polymerizing single segments $(\ell=1)$ into short polymers (say, $\ell=4$ ) frees some occupied bonds (by increasing multiple occupancies) but has little effect on the number or the length of the conducting channels. Thus, the current for $\ell=4$ is, at best, slightly enhanced over the current at $\ell=1$. However, as the polymers become longer, they also extend further, forcing particles to flow around them. Moreover, longer polymers begin to form "loops" which enclose a fair fraction of free, but now inaccessible, bonds. Hence, having a few long polymers in the system can increase the effective length of the 


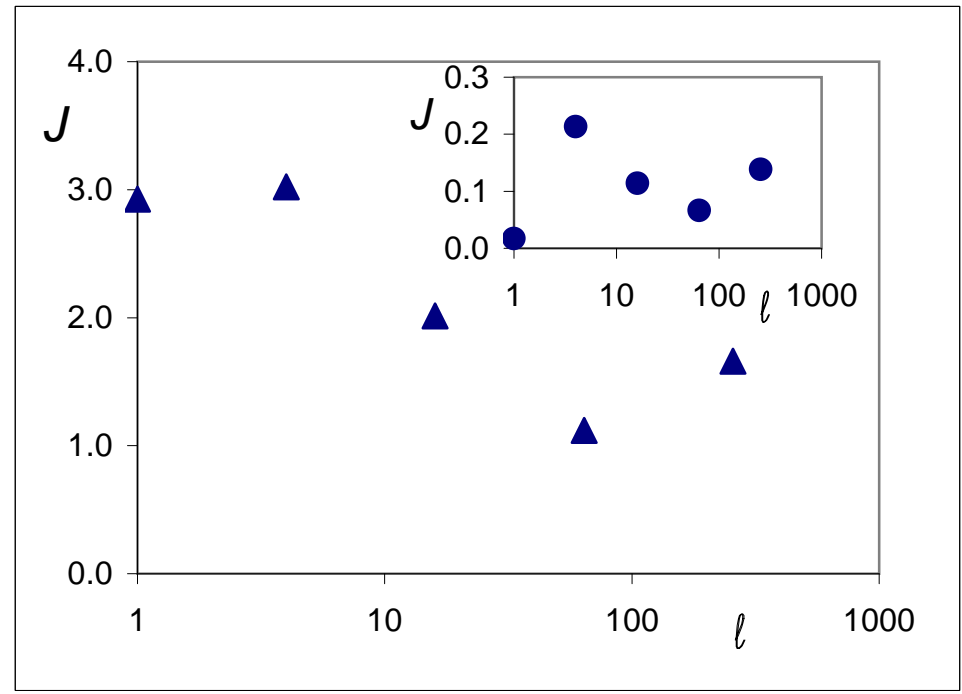

Figure 2. The gas current $J(\rho, \ell ; q)$, integrated over $100 \mathrm{MCS}$, vs. polymer length $\ell$ for $\rho=0.5$ and $\rho=0.71$ (inset), at $q=0.0$ for an $128 \times 128$ system.

conducting channels significantly and thus decrease the current, as illustrated by Fig. 2 .

Turning to the inset of Fig. 2, the picture changes drastically. This density $(\rho=0.71)$ is within a few percent of the percolation threshold, $\rho_{c}(\ell)$; if any open channels exist, they are rare and fractal. Even minor rearrangements of occupied bonds suffice to open a new channel, or block an existing one. Turning to specific $\ell$ 's, we recall that $\rho=0.71$ is slightly above the percolation threshold for single segments: $\rho_{c}(1)=0.692$. Hence, for $\ell=1$ no current should flow, at least in the thermodynamic limit. However, finite-size effects wash out a sharp percolation threshold, so that a very small current is observed: a few of the simulated polymer configurations did not percolate.

If single segments are tied together into longer polymers with, say $\ell=4$, the number of occupied bonds is lowered significantly, since higher occupancies will be generated. As a result, a much larger segment density is required for percolation: $\rho_{c}(4)=0.744$. Translated into currents, this implies that the $\ell=4$ system will carry a significant current at $\rho=0.71$, as borne out by the data shown in the inset. Yet, the tendency of polymers to form extended objects competes with this trend. We believe that the minimum, observed at $\ell=64$, is genuine (even though it lies well within the error bars) due to the fact that this system is extremely close to the percolation threshold: $\rho_{c}(64)=0.703$. If it were not for finite-size effects, we would expect the current to vanish here. To summarize, $J(0.71, \ell ; 0.0)$ is dominated by the behavior of $\rho_{c}(\ell): J$ vanishes if $\rho>\rho_{c}(\ell)$, and otherwise roughly follows $\rho_{c}(\ell)$ : the distance to the percolation threshold $\rho_{c}(\ell)$ controls the number of open channels which, in turn, control the current. Of course, there is no need to simulate systems with densities $\rho>\rho_{c}(\ell)$ since the currents are expected to vanish. 


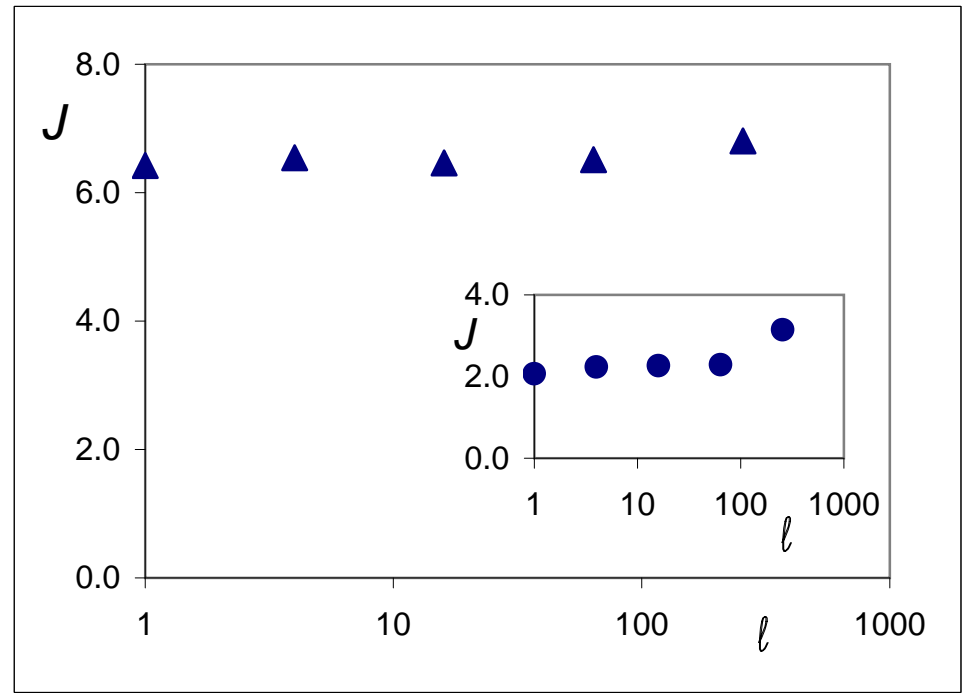

Figure 3. The gas current $J(\rho, \ell ; q)$, integrated over 100 MCS, vs. polymer length $\ell$ for $\rho=0.5$ and $\rho=1.0$ (inset), at $q=0.1$ for an $128 \times 128$ system.

Let us now compare these findings to currents measured in systems with finite energy barriers. Specifically, we choose $q=0.1$ for the rate with which a singly occupied bond is crossed by a gas molecule. For a bond occupied by $m$ segments, this rate drops to $q^{m}$. Fig. 3 shows the corresponding currents for $\rho=0.5$ and $\rho=1.0$ (inset). Both data sets show currents $J(\rho, \ell ; 0.1)$ which appear to increase gently with $\ell$. In terms of absolute values, $J(0.5, \ell ; 0.1)$ is larger than $J(1.0, \ell ; 0.1)$ because fewer bonds are occupied at the lower density. Of course, $J(0.5, \ell ; 0.1)$ is also larger than $J(0.5, \ell ; 0.0)$ : even though both cases have (on average) the same number of occupied bonds, these are blocked completely in the latter, but not the former case.

For $q=0.1$, a singly occupied bond allows gas molecules to cross in one out of 10 attempts. In order to block efficiently, a bond should carry, say, three or more segments, so that the rate of crossing is reduced to $10^{-3}$ or less. While such "highly occupied" bonds exist, of course, they are extremely unlikely to form large connected clusters, even for the largest $\ell$ : they constitute less than about $10 \%$ of bonds for $\rho=0.5$, and less than about $15 \%$ for $\rho=1.0$. As a result, there are no extended objects that might impede the flow of particles; instead, the currents are dominated by the number of completely free $(m=0)$ and singly occupied $(m=1)$ bonds. Unlike the $q=0.0$ case, domains of unoccupied bonds inside polymer loops can now be accessed and contribute to the currents. This effect is particularly stark for systems above the percolation threshold: e.g., at density $\rho=1.0$ with $q=0$, all currents would vanish.

Regarding the small dip observed in the $\rho=0.5$ data, we have no convincing explanation to offer at this stage. With statistical errors of about $10 \%$, it seems hardly significant. Yet, it is conceivable that the $J(\rho, \ell ; q)$ data reflect, in some very loose sense, the "sum" of a residual current flowing only across connected free bonds (given 
by $J(\rho, \ell ; 0.0)$ ), plus a contribution carried by the remaining (isolated) free bonds and singly occupied ones. Since the latter is likely to increase monotonically with $\ell$, while $J(0.5, \ell ; 0.0)$ is monotonically decreasing, one might interpret the dip in $J(0.5, \ell ; 0.1)$ as being the result of "adding" the two trends. Better data are needed before these questions can be settled satisfactorily.

\subsection{Solubility $M$ and first passage time $\tau$}

In very general terms, the current $J$ may be thought of as dependent on two major factors: how many particles are present inside the matrix at any given time, and how fast they move across barriers, on average. In order to quantify these, we measured the particle 'solubility' $M(\rho, \ell ; q)$ in the steady state and the mean 'first passage time' $\tau$, which is the time it takes for the first particle to emerge out of the network at its far end. If we adopt a simplistic view and imagine that the (barrier-hopping) motion of a single particle inside the polymer network is simply diffusion with an effective diffusion coefficient $D$, then the first passage time $\tau$ provides a measure of $D$ : longer time intervals $\tau$ imply lower $D$.

We find that the solubility $M(\rho, \ell ; q)$ roughly trails the current data: Higher currents are associated with larger $M(\rho, \ell ; q)$, and vice versa. Given that higher currents imply more open channels (which can then be filled with additional particles) and fewer (if any) inaccessible domains, this seems plausible. Our data for the mean first passage time in $q=0.0$ systems are unfortunately very noisy, so that no reliable conclusions can be drawn. For the $q=0.1$ systems, $\tau$ decreases with $\ell$, indicating that particles encounter fewer obstacles on their way through the network. Again, this broadly mirrors the current data.

\section{Conclusions}

In this paper, we studied a simple model of gas transport through a polymer membrane. The membrane is modeled as a network of non-interacting random walks, placed on the bonds of a square lattice in two dimensions. The network is characterized by the total segment density, $\rho$, and the length, $\ell$, of the random walks. The gas molecules are modeled as hard-core particles performing activated hopping from one lattice cell to a nearest neighbor. When they cross a bond carrying $m$ segments, they encounter an energy barrier $q^{m}$, with $q=e^{-\beta \epsilon}$. At one end of the lattice, the particle density is held at unity; at the other end, particles are immediately removed. These boundary conditions drive particles across the network. To quantify the transport properties of the network, we focus primarily on the gas current, $J(\rho, \ell ; q)$, in the steady state. We explore in particular how polymerization at fixed segment density (i.e., joining a given number of segments into polymers of varying length) affects the currents. We also measure the total number of particles stored in the network, as well as the mean first passage time for the first particle to arrive at the far end of the lattice. 
It is immediately obvious that this problem will be most interesting, and most difficult, in the low temperature regime, when the polymers form significant obstacles. Considering therefore small values of $q$, including $q=0$, our observations suggest that the transport properties of such a polymer network are affected by a competition of two main features. $(i)$ When the total segment density is fixed, the fraction of multiply occupied bonds increases since random walks frequently "retrace" their last step. As a result, polymerization generates a larger number of completely free $(m=0)$ bonds which facilitate particle motion. (ii) As the polymers increase in length, their radius of gyration also increases, as $\sqrt{\ell}$. The polymers form extended, cigar-shaped objects which may enclose domains of unoccupied bonds and block particles very efficiently, especially for $q \rightarrow 0$. As a result, for $q=0$, the current is controlled by the underlying polymer percolation problem: it is strictly zero when the polymers form a connected cluster of occupied bonds transverse to the density gradient. Exploring the associated percolation threshold, $\rho_{c}(\ell)$, as a function of $\ell$, we find that increasing the fraction of free bonds tends to shift it to higher densities while creating extended objects and "loops" pushes it towards lower densities; hence, $\rho_{c}(\ell)$ is actually non-monotonic in $\ell$. Not surprisingly, the behavior of the currents is very subtle: For $\rho=0.5$ (well below the percolation threshold), the current decreases with $\ell$, as larger polymers block more efficiently; for $\rho=0.71$, the current is non-monotonic, reflecting the behavior of $\rho_{c}(\ell)$.

For nonzero $q$, specifically $q=0.1$, the picture changes significantly. The finite extent of the polymers becomes far less important when particles can cross occupied bonds with nonzero probability. As a result, the dominant factor is now the number of bonds which allow for high particle throughput, namely, $m=0$ bonds. Since their number increases with $\ell$, the currents generally increase. Of course, the picture might become more complex again when the segment density becomes excessively large, generating a large number of "highly occupied" bonds.

To summarize, the transport properties of a random walk network are highly nontrivial. Clearly, we have explored only a small domain of the full parameter space, and our statistical errors are still large. Moreover, to reflect real polymers, more sophisticated models should surely be invoked, and more surprises may well be in store.

Acknowledgements. It is a pleasure to acknowledge fruitful discussions with E. Marand and H. Hilhorst. Over the years, both BS and RKPZ have enjoyed many hours with L. Schäfer who taught us much of what we know about polymers. This work was partially supported by NSF DMR-0088451 and DMR-0414122. GM thanks the Max Planck Gesellschaft for financial support and computational resources.

[1] E. Kesting and A.K. Fritzsche, Polymeric gas separation membranes (John Wiley and Sons, New York 1993).

[2] A. Thran, G. Kroll, F. Faupel, J. Polym. Sc. B 37, 3344 (1999).

[3] S. Kirkpatrick, Rev. Mod. Phys. 45, 574 (1973); I. Webman, Phys. Rev. Lett. 47, 1496 (1981); A. B. Harris and T. C. Lubensky, Phys. Rev. E 35, 6964 (1987); O. Stenull, H. K. Janssen and K. Oerding, Phys. Rev. E 59, 4919 (1999).

[4] Manoj Gopalakrishnan, B. Schmittmann, and R. K. P. Zia, J. Phys. A: Math. Gen. 37, L337 
(2004).

[5] H.W. Diehl and E. Eisenriegler, J. Phys. A 22, L87 (1989); F. Fougère and J. Desbois, J. Phys. A 26, 7253 (1993); L. Schäfer, Excluded Volume Effects in Polymer Solutions, as Explained by the Renormalization Group. Springer, Berlin (1999).

[6] M. F. Thorpe and P. N. Sen, J. Acoust. Soc. Am. 77, 1764 (1985); W. Xia and M. F. Thorpe, Phys. Rev. A 38, 2650 (1988). 